\title{
NORMAL BASES FOR NON-ARCHIMEDEAN SPACES OF CONTINUOUS FUNCTIONS
}

\author{
ANN VERDOODT
}

\begin{abstract}
$K$ is a complete non-archimedean valued field and $M$ is a compact, infinite, subset of $K . C(M+K)$ is the Banach space of continuous functions from $M$ to $K$, equipped with the supremum norm. Let $\left(p_{n}(x)\right)$ be a sequence of polynomials, with $\operatorname{deg} p_{n}=n$. We give necessary and sufficient conditions for $\left(p_{n}(x)\right)$ to be a normal basis for $C(M \rightarrow K)$. In the rest of the paper, $K$ contains $\mathbb{Q}_{p}$, and $V_{q}$ is the closure of the set $\left\{a q^{n} \mid n=0,1,2 \ldots\right\}$ where $a$ and $q$ are two units of $\mathbb{Z}_{p}, q$ not a root of unity. We give necessary and suffcient conditions for a sequence of polynomials $\left(r_{n}(x)\right)\left(\operatorname{deg} r_{n}=n\right.$ ) to be a normal basis for $C\left(V_{q} \rightarrow K\right)$. Furthermore, if we define $\left\{\begin{array}{l}x \\ 0\end{array}\right\}=1,\left\{\begin{array}{l}x \\ n\end{array}\right\}=\frac{(x / a-1)(x /(a q)-1) \ldots\left(x /\left(a q^{n-1}\right)-1\right)}{\left(q^{n}-1\right) \cdot(a-1)}$ if $n \geq 1$, and if $\left(j_{r}\right)$ is a sequence in $\mathbb{N}_{0}$, then we show that the sequence of polynornials $\left(\left\{\begin{array}{l}x \\ n\end{array}\right\}^{j_{n}}\right)$ forms a normal basis for $C\left(V_{Q} \rightarrow K\right)$.
\end{abstract}

\section{Introduction}

The main aim of this paper is to find normal bases for spaces of continuous functions. Therefore we start by recalling some definitions and some previous results.

Let $K$ be a non-archimedean valued field and suppose that $K$ is complete for its valuation 1. Take $M \subset K$ compact, infinite, and let $C(M \rightarrow K)$ be the Banach space of continuous functions from $M$ to $K$, equipped with the supremum norm.

Let $E$ be a non-archimedean Banach space over a non-archimedean valued field $K$. Let $e_{1}, e_{2}, \ldots$ be a finite or infinite sequence of elements of $E$. We say that this sequence is orthogonal if $\left\|\alpha_{1} e_{1}+\cdots+\alpha_{k} e_{k}\right\|=$ $\max \left\{\left\|\alpha_{i} e_{i}\right\|: i=1, \ldots, k\right\}$ for all $k$ in $\mathbb{N}$ (or for all $k$ that do not exceed the length of the sequence) and for all $\alpha_{1}, \ldots, \alpha_{k}$ in $K$. If the sequence 
is infinite, it follows that $\left\|\sum_{i=1}^{\infty} \alpha_{i} e_{i}\right\|=\max \left\{\left\|\alpha_{i} e_{i}\right\|: i=1,2, \ldots\right\}$ for all $\alpha_{1}, \alpha_{2}, \ldots$ in $K$ for which $\lim _{i \rightarrow \infty} \alpha_{i} e_{i}=0$. An orthogoal sequence $e_{1}, e_{2}, \ldots$ is called orthonormal if $\left\|e_{i}\right\|=1$ for all $i$.

This leads us to the following definition:

If $E$ is a non-archimedean Banach space over a non-archimcdean valued field $K$, then a family $\left(e_{i}\right)$ of elements of $E$ is a (ortho)normal basis of $E$ if the family $\left(e_{i}\right)$ is orthonormal and also a basis.

An equivalent formulation is

If $E$ is a non-archimedean Banach space over a non-archimedean valued field $K$, then a family $\left(e_{i}\right)$ of elements of $E$ is a (ortho)normal basis of $E$ if each element $x$ of $E$ has a unique representation $x=\sum_{i} x_{i} e_{i}$ where $x_{i} \in K$ and $x_{i} \rightarrow 0$ if $i \rightarrow \infty$, and if the norm of $x$ is the supremum of the norms of $x_{i}$.

In $[6$, chapter 5, 5.27 and 5.33] we find the following theorem which is due to $Y$. Amice:

\section{Theorem 1.}

Let $K$ be a non-archimedean valued field, complete with respect to its norm $|$.$| , and let M$ be a compact, infinite subset of $K$.

Let $\left(u_{n}\right)$ be an injective sequence in $M$.

Define $p_{0}(x)=1, p_{n}(x)=\left(x-u_{n-1}\right) p_{n-1}(x)$ for $n \geq 1, q_{n}(x)=$ $\frac{p_{n}(x)}{p_{n}\left(u_{n}\right)}$.

Then $\left(q_{n}(x)\right)$ forms a normal basis for $C(M \rightarrow K)$ if and only if $\left\|q_{n}\right\|=1(\forall n)$.

If $\left(q_{n}(x)\right)$ forms a normal basis for $C(M \rightarrow K)$ and $f$ is an element of $C(M \rightarrow K)$, then

$$
f(x)=\sum_{n=0}^{\infty} a_{n} q_{n}(x) \text { where } a_{n}=p_{n}\left(u_{n}\right) \sum_{i=0}^{n} \frac{f\left(u_{i}\right)}{p_{n+1}^{\prime}\left(u_{i}\right)}
$$

We remark that there always exist sequences $\left(q_{n}(x)\right)$ such that $\left\|q_{n}\right\|=1$ for all $n$.

We will call a sequence of polynomials $\left(p_{n}(x)\right)$ a polynomial sequence if $p_{n}$ is exactly of degree $n$ for all $n$.

After all these definitions, we now give a survey of the results in this article. 
In Section 2 of this paper, $K$ is a non-archimedean complete field, and $M$ is a compact, infinite subset of $K$. In Theorems 2 and 3 we will give necessary and sufficient conditions for a polynomial sequence $\left(p_{n}(x)\right)$ to be a normal basis for $C(M \rightarrow K)$.

In Sections $3,4,5$ and 6 we consider the following situation: $\mathbb{Z}_{p}$ is the ring of $p$-adic integers, $\mathbb{Q}_{p}$ the field of $p$-adic numbers, and $K$ is a non-archimedean valued field, $K$ containing $\mathbb{Q}_{p}$, and we supppose that $K$ is complete for the valuation |.|, which extends the $p$-adic valuation. Let $a$ and $q$ be two units of $\mathbb{Z}_{p}, q$ not a root of unity. We define $V_{q}$ to be the closure of the set $\left\{a q^{n} \mid n=0,1,2, \ldots\right\}$. A description of the set $V_{q}$ will be given in Section 3 (Lemmas 4 and 5 ). In Section 4 , Theorem 4 , we will give necessary and sufficient conditions for a polynomial sequence $\left(r_{n}(x)\right)$ to be a normal basis for $C\left(V_{q} \rightarrow K\right)$.

If we put $\left\{\begin{array}{l}x \\ 0\end{array}\right\}=1,\left\{\begin{array}{l}x \\ n\end{array}\right\}=\frac{(x / a-1)(x /(a q)-1) \ldots\left(x /\left(a q^{n-1}\right)-1\right)}{\left(q^{n}-1\right) \ldots(q-1)}$ if $n \geq 1$, and if $\left(j_{n}\right)$ is a sequence in $\mathbb{N}_{0}$, then we show in Theorem 5 of Section 5 that $\left(\left\{\begin{array}{l}x \\ n\end{array}\right\}^{j_{n}}\right)$ forms a normal basis for $C\left(V_{q} \rightarrow \mathbb{Q}_{p}\right)$. The proof we give here is only valid when we work with a discrete valuation.

In Section 6 , Theorem 6 we show that $\left(\left\{\begin{array}{l}x \\ n\end{array}\right\}^{j_{n}}\right)$ also forms a normal basis for $C\left(V_{q} \rightarrow K\right)$, where the valuation of $K$ does not have to be discrete, as was the case in the previous section.

To prove this, we need the results of Section 5 .

S. Caenepeel ([3]) proved the following: Let $\left(\begin{array}{l}x \\ n\end{array}\right)=\frac{x(x-1) \ldots(x-n+1)}{n !}$ if $n \geq 1,\left(\begin{array}{l}x \\ 0\end{array}\right)=1$ (the binomial polynomials), then for each $s \in \mathbb{N}_{0},\left(\left(\begin{array}{l}x \\ n\end{array}\right)^{s}\right)$ forms a normal basis for $C\left(\mathbb{Z}_{p} \rightarrow \mathbb{Q}_{p}\right)$, and each function $f$ in $C\left(\mathbb{Z}_{p} \rightarrow\right.$ $\mathbb{Q}_{p}$ ) can be written as a uniformly convergent series

$$
f(x)=\sum_{n=0}^{\infty} a_{n}^{(s)}\left(\begin{array}{l}
x \\
n
\end{array}\right)^{s}
$$

where

$$
a_{n}^{(s)}=\sum_{k=0}^{n}(-1)^{n-k}\left(\begin{array}{l}
n \\
k
\end{array}\right)^{s} f(k) \alpha_{n-k}^{(s)}
$$

and

$$
\alpha_{m}^{(s)}=\sum_{\substack{\left(j_{1}, \ldots, j_{r}\right) \\
\sum j_{i}=m_{i}, j \leq j_{i} \leq m}}^{\alpha_{0}^{(s)}=1}(-1)^{r+m}\left(\begin{array}{c}
m \\
j_{1} \ldots j_{r}
\end{array}\right)^{s}
$$


If $\left(j_{n}\right)$ is a sequence in $\mathbb{N}_{0}$, then the sequence of polynomials $\left(\left(\begin{array}{l}x \\ n\end{array}\right)^{j_{n}}\right)$ also forms a normal basis of $C\left(\mathbb{Z}_{p} \rightarrow \mathbb{Q}_{p}\right)([4, \mathrm{p} .158])$.

Now we can find an analogous result on the space $C\left(V_{q} \rightarrow K\right)$ : each function $f$, element of $C\left(V_{q} \rightarrow K\right)$, can be written as a uniformly convergent series

$$
f(x)=\sum_{n=0}^{\infty} b_{n}^{(s)}\left\{\begin{array}{l}
x \\
n
\end{array}\right\}^{s}
$$

and we can give an expression for the coefficients $b_{n}^{(s)}$, which is analogous to the expression in (1). This result can be found in Proposition 1 of Section 6.

Acknowledgement. I want to thank Professor Van Hamme and Professor Caenepeel for the advice and the help they gave me during the preparation of this paper.

\section{Normal bases for $C(M \rightarrow K)$}

In this section, $K$, is a non-archimedean valued field, complete with respect to his norm |.|, and $M$ is a compact, infinite subset of $K$.

Before we generalize Amice's Theorem, we give a lemma.

\section{Lemma 1.}

Let $\left(u_{n}\right)$ be an injective sequence in $M$, and let $q_{0}(x)=1, q_{n}(x)=$ $\frac{\left(x-u_{0}\right) \ldots\left(x-u_{n-1}\right)}{\left(u_{n}-u_{0}\right) \ldots\left(u_{n}-u_{n-1}\right)}$ for $n \geq 1$, where $\left\|q_{n}\right\|=1$ for all $n$.

If $p(x)$ is a polynomial in $k[x]$ of degree $n$, then there exists an index $i, 0 \leq i \leq n$, such that $\|p\|=\left|p\left(u_{i}\right)\right|$.

Proof:

There exist coefficients $c_{j}$ such that $p(x)=\sum_{j=0}^{n} c_{j} q_{j}(x)$. Now suppose that $\left|p\left(u_{i}\right)\right|<\|p\|$ for all $i, 0 \leq i \leq n$. This will lead to a contradiction.

Supposing that $\left|p\left(u_{i}\right)\right|<\|p\|$ for all $i, 0 \leq i \leq n$, we will prove by induction that $\left|c_{i}\right|<\|p\|$ for $0 \leq i \leq n$.

Now $p\left(u_{0}\right)=\sum_{j=0}^{n} c_{j} q_{j}\left(u_{0}\right)=c_{0}$, so $\left|c_{0}\right|<\|p\|$.

Further, $p\left(u_{1}\right)=\sum_{j=0}^{n} c_{j} q_{j}\left(u_{1}\right)=c_{0}+c_{1}$, so $\left|c_{0}+c_{1}\right|<\|p\|$, and combining this with the previous we find $\left|c_{1}\right|<\|p\|$.

Suppose we already have that $\left|c_{i}\right|<\|p\|$ for $0 \leq i<k \leq n$. 
Then $p\left(u_{k}\right)=\sum_{j=0}^{n} c_{j} q_{j}\left(u_{k}\right)=\sum_{j=0}^{k-1} c_{j} q_{j}\left(u_{k}\right)+c_{k}$, so $\left|\sum_{j=0}^{k-1} c_{j} q_{j}\left(u_{k}\right)+c_{k}\right|<$ $\|p\|$. Since $\left|c_{i}\right|<\|p\|$ for $0 \leq i<k \leq n$ and since $\left\|q_{j}\right\|=1$, we find that $\left|c_{k}\right|<\|p\|$.

So we may conclude that $\left|c_{i}\right|<\|p\|$ for $0 \leq i \leq n$.

But then we have, since $\left(q_{n}(x)\right)$ forms a normal basis (Theorem 1), $\|p\|=\max _{0 \leq i \leq n}\left\{\left|c_{i}\right|\right\}<\|p\|$ which is clearly a contradiction.

Since $\left|p\left(u_{i}\right)\right| \leq\|p\|$, we may conclude that there exists an index $i, 0 \leq$ $i \leq n$, such that $\|p\|=\left|p\left(u_{i}\right)\right|$.

Procecding from the theorem of Amice, we can marke more normal bases with the following theorem:

\section{Theorem 2.}

Let $\left(q_{n}(x)\right)$ be a normal basis as found in Theorem 1 .

Define $p_{n}(x)=\sum_{j=0}^{n} c_{n ; j} q_{j}(x), c_{n ; j} \in K, c_{n: n} \neq 0$.

Then $\left(p_{n}(x)\right)$ forms a normal basis for $C(M \rightarrow K)$ if and only if $\left\|p_{n}\right\|=1$ and $\left|c_{n ; n}\right|=1$ for all $n$.

\section{Proof:}

Suppose that the sequence $\left(p_{n}(x)\right)$ forms a normal basis for $C(M \rightarrow$ K).

It is clear that the norm of $p_{n}$ must equal one. Since $\left(q_{n}(x)\right)$ forms a normal basis, this implies that $\left|c_{n ; n}\right| \leq 1$.

There exist coefficients $d_{n ; j}$ such that $q_{n}=\sum_{j=0}^{n} d_{n ; j} P_{j}(x)$ and so wc have $1=\left\|q_{n}\right\|=\max _{0 \leq j \leq n}\left\{\left|d_{n ; j}\right|\right\}$ so $\left|d_{n ; n}\right| \leq 1$.

Further, $q_{n}=\sum_{j=0}^{n} d_{n ; j} p_{j}(x)=\sum_{j=0}^{n} d_{n ; j} \sum_{i=0}^{j} c_{j ; i} q_{i}=\sum_{i=0}^{n} q_{i} \sum_{j=i}^{n} d_{n ; j} c_{j ; i}$ and this implies $d_{n ; n} c_{n ; n}=1$.

Combining this with the fact that $\left|d_{n ; n}\right| \leq 1$ and $\left|c_{n ; n}\right| \leq I$, we conclude $\left|d_{n ; n}\right|=1$ and $\left|c_{n ; n}\right|=1$.

We now prove the other implication.

Let $k$ be an arbitrary element of $\mathbb{N}$ and let $b_{0}, b_{1}, \ldots, b_{k}$ be arbitrary elements of $K$. For the orthonormality of the sequence $\left(p_{n}(x)\right)$, we have to show

$$
\left\|b_{0} p_{0}+\cdots+b_{k} p_{k}\right\|=\max _{0 \leq n \leq k}\left\{\left\|b_{i} p_{i}\right\|\right\}=\max _{0 \leq n \leq k}\left\{\left|b_{i}\right|\right\}
$$


If $\max _{0 \leq n \leq h}\left\{\left|b_{n}\right|\right\}=0$ there is nothing to prove.

If $\max _{0 \leq n \leq k}\left\{\left|b_{n}\right|\right\}>0$, then put $I=\left\{n|0 \leq n \leq k|\left|b_{n}\right|=\max _{0 \leq j \leq k}\left\{\left|b_{j}\right|\right\}\right\}$.

There exists an $N$ such that $N=\max \{i \in I\}$.

We have $\left|\sum_{n=0}^{k} b_{n} p_{n}(x)\right| \leq \max _{0 \leq n \leq k}\left\{\left|b_{n} p_{n}(x)\right|\right\} \leq\left|b_{N}\right|$, and so $\left\|\sum_{n=0}^{k} b_{n} p_{n}\right\| \leq \max _{0 \leq n \leq k}\left\{\left|b_{n}\right|\right\}$.

Put $\sum_{n=0}^{k} b_{n} p_{n}(x)=\sum_{n=0}^{N} b_{n} p_{n}(x)+\sum_{n=N+1}^{k} b_{n} p_{n}(x)=\hat{f}(x)+\hat{f}(x)$, where we have $\|\tilde{f}\| \leq \max _{0 \leq n \leq k}\left\{\left|b_{n}\right|\right\},\|\hat{f}\|<\max _{0 \leq n \leq k}\left\{\left|b_{n}\right|\right\}$ (strict inequality).

$$
\begin{aligned}
\tilde{f}(x) & =\sum_{n=0}^{N} b_{n} p_{n}(x)=\sum_{n=0}^{N} b_{n} \sum_{j=0}^{n} c_{n ; j} q_{j}(x)=\sum_{j=0}^{N} q_{j}(x) \sum_{n=j}^{N} b_{n} c_{n ; j} \\
& =\sum_{j=0}^{N-1} q_{j}(x) \sum_{n=j}^{N} b_{n} c_{n ; j}+q_{N}(x) b_{N} c_{N ; N} .
\end{aligned}
$$

We distinguish two cases:

$$
\text { a) }\left\|\sum_{j=0}^{N-1} q_{j} \sum_{n=j}^{N} b_{n} c_{n ; j}\right\|<\left|b_{N}\right| \text {. }
$$

Since $\left|q_{N}\left(u_{N}\right) b_{N} c_{N_{i} N}\right|=\left|b_{N}\right|$, it follows that $\left|\tilde{f}\left(u_{N}\right)\right|=\left|b_{N}\right|$, and so $\left\|\sum_{n=0}^{k} b_{n} p_{n}\right\|=\left|b_{N}\right|=\max _{0 \leq n \leq k}\left\{\left|b_{n}\right|\right\}$.
b) $\left\|\sum_{j=0}^{N-1} q_{j} \sum_{n=j}^{N} b_{n} c_{n ; j}\right\|=\left|b_{N}\right|$

There exists an $i, 0 \leq i \leq N-1$, such that $\left|\sum_{j=0}^{N-1} q_{j}\left(u_{i}\right) \sum_{n=j}^{N} b_{n} c_{n ; j}\right|=\left|b_{N}\right|$ (Lemma 1).

Then we have $\left|\vec{f}\left(u_{i}\right)=\right| \sum_{j=0}^{N-1} q_{j}\left(u_{i}\right) \sum_{n=j}^{N} b_{n} c_{n ; j}|=| b_{N} \mid$, and so $\left\|\sum_{n=0}^{k} b_{n} p_{n}\right\|=\left|b_{N}\right|=\max _{0 \leq n \leq k}\left\{\left|b_{n}\right|\right\}$.

We conclude that the sequence $\left(p_{n}(x)\right)$ is orthonormal.

By [6, p. 165, Lemma 5.1] and by Kaplansky's Theorem (see e.g. [6, p. 191 , Theorem 5.28]) it follows that $\left(p_{n}(x)\right)$ forms a basis of $C(M \rightarrow K)$, since the $k$ linear span of the polynomials $p_{n}(x)$ contains $K[x]$. 


\section{Theorem 3.}

Let $\left(p_{n}(x)\right)$ be a polynomial sequence in $K[x]$, which forms a normal basis for $C(M \rightarrow K)$, and let $\left(r_{n}(x)\right)$ be a polynomial sequence in $K[x]$ such that $r_{n}(x)=\sum_{j=0}^{n} e_{n_{i j}} p_{j}(x), e_{n ; j} \in K$. Then the following are equivalent:

i) $\left(r_{n}(x)\right)$ forms a normal basis for $C(M \rightarrow K)$

ii) $\left\|r_{n}\right\|=1,\left|e_{n ; n}\right|=1$

iii) $\left|e_{n ; j}\right| \leq 1,\left|e_{n ; n}\right|=1$.

\section{Proof:}

i) $\Leftrightarrow$ ii) follows from Theorem 2, using the expression $p_{n}(x)=$ $\sum_{j=0}^{n} c_{n ; j} q_{j}(x)$, and ii) $\Leftrightarrow$ iii) follows from the fact that, $\left(p_{n}(x)\right)$ forms a normal basis.

\section{The set $V_{q}$}

From now on, $K$ is a non-archimedean valued field, $K$ contains $\mathbb{Q}_{p}$, and $K$ is complete for the valuation |.], which extends the $p$-adic valuation.

The aim now is to find normal bases for the space $C\left(V_{q} \rightarrow K\right)$. Therefore, we start by giving a description of the set $V_{q}$ (Lemmas 4 and 5 below).

\section{Definition.}

If $b$ is an element of $\mathbb{Z}_{p}, b \equiv 1(\bmod p), x$ an element of $\mathbb{Z}_{p}$, then we put $b^{x}=\lim _{n \rightarrow x} b^{n}$. The mapping: $\mathbb{Z}_{p} \rightarrow \mathbb{Z}_{p}: x \rightarrow b^{x}$ is continuous.

For more details, we refer the reader to $[4$, Section 32$]$.

\section{Lemma 2.}

Let $\alpha$ be an element of $\mathbb{Z}_{p}, \alpha \equiv 1\left(\bmod p^{r}\right), \alpha \neq \equiv 1\left(\bmod p^{r+1}\right) r \geq 1$. If $(p, r) \neq(2,1), \beta \in \mathbb{Z}_{p} \backslash\{0\}$ then

$$
\begin{aligned}
& \alpha^{\beta} \equiv\left(\bmod p^{r+\operatorname{ord}_{p} \beta}\right) \\
& \alpha^{\beta} \not \equiv 1\left(\bmod p^{r+1+\operatorname{ord}_{p} \beta}\right) .
\end{aligned}
$$

Proof:

Let $\alpha=1+\gamma p^{r}$, and let $\gamma=\gamma_{0}+\gamma_{1} p+\ldots$, with $\gamma_{0} \neq 0$, be the Henseldevelopment of the $p$-adic integer $\gamma([4$, Section 3$])$. 
Then we have

$$
\begin{aligned}
\alpha^{p} & =\left(1+\gamma p^{r}\right)^{p}=\sum_{k=0}^{p}\left(\begin{array}{l}
p \\
k
\end{array}\right)\left(\gamma p^{r}\right)^{k}=1+p \gamma p^{r}+\ldots\left(\gamma p^{r}\right)^{p} \\
& =1+\gamma_{0} p^{r+1} \ldots(\text { remark }: r+1 \neq r p),
\end{aligned}
$$

and so $\alpha^{p} \equiv 1\left(\bmod p^{r+1}\right), \alpha^{p} \not \equiv 1\left(\bmod p^{r+2}\right)$.

If we continue in this way, we find: $\alpha^{p^{s}} \equiv 1\left(\bmod p^{r+s}\right), \alpha^{p^{s}} \neq \equiv 1(\bmod$ $\left.p^{r+1+s}\right)$.

Now take $k$ such that $2 \leq k \leq p-1$.

$$
\alpha^{k}=\left(1+\gamma p^{r}\right)^{k}=\sum_{j=0}^{k}\left(\begin{array}{l}
k \\
j
\end{array}\right)\left(\gamma p^{r}\right)^{j}=1+k \gamma p^{r}+\ldots\left(\gamma p^{r}\right)^{k} .
$$

$k \gamma$ cannot be a multiple of $p$, since neither $k$ or $\gamma$ is divisible by $p$.

So $\alpha^{k} \equiv 1\left(\bmod p^{r}\right), \alpha^{k} \not 1\left(\bmod p^{r+1}\right)$.

Let $n$ be an element of $\mathbb{N}_{0}$. If we combine the previous results then we find $\alpha^{n} \equiv 1\left(\bmod p^{r+\operatorname{ord}_{p} n}\right), \alpha^{n} \not \equiv 1\left(\bmod p^{r+1+\operatorname{ord}_{p} n}\right)$.

The lemma follows by continuity.

\section{Lemma 3.}

Let $\alpha$ be an element of $\mathbb{Z}_{2}, \alpha \equiv 3(\bmod 4)$. Define a natural number $n$ by $\alpha=1+2+2^{2} \varepsilon, \varepsilon=\varepsilon_{0}+\varepsilon_{1} 2+\varepsilon_{2} 2^{2}+\ldots, \varepsilon_{0}=\varepsilon_{1}=\cdots=\varepsilon_{n-1}=1$, $\varepsilon_{n}=0$.

If $\beta \in \mathbb{Z}_{2} \backslash\{0\}$, ord $\beta=0$ then

$$
\begin{aligned}
& \alpha^{\beta} \equiv 1(\bmod 2) \\
& \alpha^{\beta} \not \equiv 1(\bmod 4) .
\end{aligned}
$$

If $\beta \in \mathbb{Z}_{2} \backslash\{0\}, \operatorname{ord}_{2} \beta=k \geq 1$ then

$$
\begin{aligned}
& \alpha^{\beta} \equiv 1\left(\bmod 2^{n+2+\operatorname{ord}_{2} \beta}\right) \\
& \alpha^{\beta} \equiv 1\left(\bmod 2^{n+3+\operatorname{ord}_{2} \beta}\right) .
\end{aligned}
$$

\section{Proof:}

$$
\alpha=3+4 \varepsilon \text {. Hence } \alpha^{2}=1+2^{3}(1+\varepsilon)(1+2 \varepsilon) \text {. }
$$

Since $\varepsilon=\varepsilon_{0}+\varepsilon_{1} 2+\varepsilon_{2} 2^{2}+\ldots, \varepsilon_{0}=\varepsilon_{1}=\cdots=\varepsilon_{n-1}=1, \varepsilon_{n}=0$, $\operatorname{ord}_{2}(1+2 \varepsilon)=0$, wc have $\alpha^{2} \equiv 1\left(\bmod 2^{n+3}\right), \alpha^{2} \not \equiv 1\left(\bmod 2^{n+4}\right)$. 
Then

$$
\begin{aligned}
\alpha^{2^{k+1}}=\left(\alpha^{2}\right)^{2^{k}} & \equiv 1\left(\bmod 2^{n+3+k}\right) \\
& \neq 1\left(\bmod 2^{n+4+k}\right) \text { by Lemma } 2(k \geq 1) .
\end{aligned}
$$

So $\alpha^{2^{k}} \equiv 1\left(\bmod 2^{n+2+k}\right), \alpha^{2^{k}} \not \equiv 1\left(\bmod 2^{n+3+k}\right)(k \geq 1)$.

In an analogous way as in the previous lemma, we show

If $s \in \mathbb{N}_{0}$, ord $s=0$, then $\alpha^{s} \equiv 1(\bmod 2), \alpha^{s} \not \equiv 1(\bmod 4)$.

If $s \in \mathbb{N}_{0}, \operatorname{ord}_{2} s=k \geq 1$, then $\alpha^{s} \equiv 1\left(\bmod 2^{n+2+o r d} s\right), \alpha^{s} \not \equiv I(\bmod$ $2^{n+3+\operatorname{ord}_{2} s}$ ).

The lemma follows by continuity.

In the following lemma, $m$ is the smallest integer such that $q^{m} \equiv 1$ $(\bmod p) .($ Remark: $1 \leq m \leq p-1)$.

\section{Lemma 4.}

Let $q^{m} \equiv 1\left(\bmod p^{k_{0}}\right), q^{m} \not \equiv 1\left(\bmod p^{k_{0}+1}\right)$.

If $\left(p, k_{0}\right) \neq(2,1)$, then $V_{q}=\underset{0 \leq r \leq m-1}{\bigcup}\left\{x \in \mathbb{Z}_{p}|| x-a q^{r} \mid \leq p^{-k_{0}}\right\}$.

Proof:

We take the $m$ balls $\left\{x \in \mathbb{Z}_{p}|| x-a q^{r} \mid \leq p^{-k_{0}}\right\}, 0 \leq r \leq m-1$.

Every element $a q^{r}\left(q^{m}\right)^{n}(0 \leq r \leq m-1, n \in \mathbb{N})$ belongs to one of these balls: $\left|a q^{r}\left(q^{7 n}\right)^{n}-a q^{r}\right|=\left|a q^{r}\right|\left|\left(q^{m n}\right)^{n}-1\right| \leq p^{-k_{0}}$ (Lemma 2).

Since $V_{q}$ is the closure of $\left\{a q^{n} \mid n=0,1,2, \ldots\right\}=\left\{a q^{r}\left(q^{m}\right)^{n} \mid 0 \leq r \leq\right.$ $m-1, n \in \mathbb{N}\}$, we have that $\underset{0 \leq r \leq m-1}{\bigcup}\left\{x \in \mathbb{Z}_{p}|| x-a q^{r} \mid \leq p^{-k_{0}}\right\} \supset V_{q}$.

The $m$ balls $\left\{x \in \mathbb{Z}_{p}|| x-a q^{T} \mid \leq p^{-k_{0}}\right\}$ are pairwise disjoint: take $r, s \in$ $\{0,1, \ldots, m-1\}, r \neq s$ e.g. $r>s$, then $\left|a q^{r}-a q^{s}\right|=\left|a q^{s}\right|\left|q^{r-s}-1\right|=1$.

We remark that it is impossible to take balls with a smaller radius: $\left|a q^{T}-a q^{T} q^{m}\right|=\left|a q^{r}\right|\left|1-q^{m}\right|=p^{-k_{0}}$.

Let $r$ be fixed: $\left\{x \in \mathbb{Z}_{p}|| x-a q^{r} \mid \leq p^{-k_{0}}\right\}$.

We take the following $p$ elements of $V_{q}: a q^{r}\left(q^{m}\right)^{0}, a q^{r}\left(q^{m}\right)^{1}, \ldots$, $a q^{r}\left(q^{m}\right)^{p-1}$.

Each of these elements belongs to $\left\{x \in \mathbb{Z}_{p}|| x-a q^{r} \mid \leq p^{-k_{0}}\right\}$ : $\left|a q^{r}\left(q^{m}\right)^{i}-a q^{r}\right|=\left|a q^{r}\right|\left|\left(q^{m}\right)^{i}-1\right| \leq p^{-k_{0}}$ by Lemma $2(0 \leq i \leq p-1)$.

Furthermore, if $i, j \in\{0,1, \ldots, p-1\}, i \neq j$, say $i>j$, then $\left|a q^{r}\left(q^{m}\right)^{i}-a q^{r}\left(q^{m}\right)^{j}\right|=\left|a q^{r}\left(q^{m}\right)^{j}\right|\left|\left(q^{m}\right)^{i-j}-1\right|=p^{-k_{0}}$ by Lemma 2 since $0<i-j \leq p-1$.

So these $p$ elements define $p$ disjoint balls with radius $p^{-\left(k_{0}+1\right)}$ which cover $\left\{x \in \mathbb{Z}_{p}|| x-a q^{r} \mid \leq p^{-k_{0}}\right\}$. 
We take $\left\{x \in \mathbb{Z}_{p}|| x-a q^{r}\left(q^{m}\right)^{i} \mid \leq p^{-\left(k_{0}+1\right)}\right\}, i \in\{0,1, \ldots, p-1\}, i$ fixed.

Take the $p$ elements $a q^{r}\left(q^{m}\right)^{i+j p}, 0 \leq j \leq p-1$.

These elements belong to $\left\{x \in \mathbb{Z}_{p}|| x-a q^{r}\left(q^{m}\right)^{i} \mid \leq p^{-\left(k_{0}+1\right)}\right\}$ : $\left|a q^{r}\left(q^{m}\right)^{i+j p}-a q^{r}\left(q^{m}\right)^{i}\right|=\left|a q^{r}\left(q^{m}\right)^{i}\right|\left|\left(q^{m}\right)^{j p}-1\right| \leq p^{-\left(k_{0}+1\right)}$ by Lemma. $2(0 \leq j \leq p-1)$.

Furthermore, if $j, k \in\{0,1, \ldots, p-1\}, k \neq j$, say $k>j$, then $\left|a q^{r}\left(q^{m}\right)^{i+k p}-a q^{r}\left(q^{m}\right)^{i+j p}\right|=\left|a q^{r}\left(q^{m}\right)^{i+j p}\right|\left|\left(q^{m}\right)^{(k-j) p}-1\right|=p^{-\left(k_{0}+1\right)}$ by Lemma 2 since $0<k-j \leq p-1$. So these $p$ elements defino $p$ disjoint balls with radius $p^{-\left(k_{0}+2\right)}$ which cover $\left\{x \in \mathbb{Z}_{p}|| x-a q^{r}\left(q^{m}\right)^{i} \mid \leq\right.$ $\left.p^{-\left(k_{0}+1\right)}\right\}$.

We can continue this way.

Suppose we have $\left\{x \in \mathbb{Z}_{p}|| x-a q^{r}\left(q^{m}\right)^{i_{0}+i_{1} p+\cdots+i_{n} p^{n}} \mid \leq p^{-\left(k_{0}+n+1\right)}\right\}$, $i_{0}, i_{1}, \ldots, i_{n} \in\{0,1, \ldots, p-1\}, i_{0}, i_{1}, \ldots, i_{n}$ fixed.

We take the $p$ elements $a q^{r}\left(q^{m}\right)^{i_{0}+i_{1} p+i_{n} p^{n}+i_{n+1} p^{n+1}}, 0 \leq i_{n+1} \leq p-1$.

All these elements belong to $\left\{x \in \mathbb{Z}_{p}|| x-a q^{r}\left(q^{m}\right)^{i_{0}+i_{1} p+\cdots+i_{n} p^{n}} \mid \leq\right.$ $\left.p^{-\left(k_{0}+n+1\right)}\right\}$ :

$$
\begin{aligned}
& \left|a q^{r}\left(q^{m}\right)^{i_{0}+i_{1} p+\cdots+i_{2} p^{n}+i_{n+1} p^{n+1}}-a q^{r}\left(q^{m}\right)^{i_{0}+i_{1} p+\cdots+i_{n} p^{n}}\right| \\
& \quad=\left|a q^{r}\left(q^{m}\right)^{i_{0}+i_{1} p+\cdots+i_{n} p^{n}}\right|\left|a q^{r}\left(q^{m}\right)^{i_{n+1} p^{n+1}}-1\right| \leq p^{-\left(k_{0}+n+1\right)} .
\end{aligned}
$$

Furthermore, if $j, k \in\{0,1, \ldots, p-1\}, k \neq j$, say $k>j$, then

$$
\begin{aligned}
& \left|a q^{r}\left(q^{m}\right)^{i_{0}+i_{1} p+\cdots+i_{n} p^{n}+k p^{n+1}}-a q^{T}\left(q^{m}\right)^{i_{0}+i_{1} p+\cdots+i_{n} p^{n}+j p^{n+1}}\right| \\
& \quad=\left|a q^{r}\left(q^{m}\right)^{i_{0}+i_{1} p+\cdots+i_{n} p^{n}+j p^{n+1}}\right|\left|\left(q^{m}\right)^{(k-j) p^{n+1}}-1\right|=p^{-\left(k_{0}+n+1\right)} .
\end{aligned}
$$

So these $p$ elements define $p$ disjoint balls with radius $p^{-\left(k_{0}+n+2\right)}$ which cover $\left\{x \in \mathbb{Z}_{p}|| x-a q^{T}\left(q^{m}\right)^{i_{0}+i_{1} p+\cdots+i_{n} p^{n}} \mid \leq p^{-\left(k_{0}+n+1\right)}\right\}$.

Continuing this way, we find closed balls with radius tending to zero and whose centers are elements of $\left\{a q^{n} \mid n=0,1,2, \ldots\right\}$, and these balls cover $\bigcup_{0 \leq r \leq m-1}\left\{x \in \mathbb{Z}_{p}|| x-a q^{r} \mid \leq p^{-k_{0}}\right\}$. So $\underset{0 \leq r \leq m-1}{\bigcup}\left\{x \in \mathbb{Z}_{p}|| x-\right.$ $\left.a q^{r} \mid \leq p^{-\bar{k}_{0}}\right\}$ is the closure of $\left\{a q^{n} \mid n=0,1,2, \ldots\right\}$. But this means that $V_{q}=\underset{0 \leq r \leq m-1}{\bigcup_{0}}\left\{x \in \mathbb{Z}_{p}|| x-a q^{r} \mid \leq p^{-k_{0}}\right\}$.

\section{Lemma 5.}

Let $q \equiv 3(\bmod 4)$.

Then $V_{q}=\left\{x \in \mathbb{Z}_{2}|| x-a \mid \leq 2^{-(N+3)}\right\} \cup\left\{x \in \mathbb{Z}_{2}|| x-a q \mid \leq 2^{-(N+3)}\right\}$, where $q=1+2+2^{2} \varepsilon_{,} \varepsilon=\varepsilon_{0}+\varepsilon_{1} 2+\varepsilon_{2} 2^{2}+\ldots, \varepsilon_{0}=\varepsilon_{1}=\cdots=\varepsilon_{N-1}=1$, $\varepsilon_{N}=0$. 
Proof:

Every element $a q^{n}$ belongs to $\left\{x \in \mathbb{Z}_{2}|| x-a \mid \leq 2^{-(N+3)}\right\} \cup\{x \in$ $\left.\mathbb{Z}_{2}|| x-a q \mid \leq 2^{-(N+3)}\right\}:\left|a q^{0+2 k}-a\right|=|a|\left|q^{2 k}-1\right| \leq 2^{-(N+3)}$ and $\left|a q^{1+2 k}-a q\right|=|a q|\left|q^{2 k}-1\right| \leq 2^{-(N+3)}$ by Lemma $3(k \in \mathbb{N})$. Since $V_{q}$ is the closure of $\left\{a q^{n} \mid n=0,1,2, \ldots\right\}$, we have that $\left\{x \in \mathbb{Z}_{2}|| x-a \mid \leq\right.$ $\left.2^{-(N+3)}\right\} \cup\left\{x \in \mathbb{Z}_{2}|| x-a q \mid \leq 2^{-(N+3)}\right\} \supset V_{q}$.

The balls $\left\{x \in \mathbb{Z}_{2}|| x-a \mid \leq 2^{-(N+3)}\right\}$ and $\left\{x \in \mathbb{Z}_{2}|| x-a q \mid \leq 2^{-(N+3)}\right\}$ are disjoint: $|a q-a|=|a||q-1|=2^{-1}$.

We remark that it is impossible to take balls with a smaller radius: $\left|a q^{2}-a\right|=|a|\left|q^{2}-1\right|=2^{-(N+3)}$ and $\left|a q^{1+2}-a q\right|=|a q|\left|q^{2}-1\right|=2^{-(N+3)}$ (Lemma 3).

From now on, we can prove the lemma in an analogous way as Lemma 4.

We will need these lemmas in the sequel.

\section{Normal bases for $C\left(V_{q} \rightarrow K\right)$}

We want to give a thcorem analogous to Theorem 3 , but with $C(M \rightarrow$ $K)$ replaced by $C\left(V_{q} \rightarrow K\right)$.

Therefore, we need some notations.

We introduce the following:

$[n] !=[n][n-1] \ldots[1],[0] !=1$, where $[n]=\frac{q^{n}-1}{q-1}$ if $n \geq 1$.

$\left[\begin{array}{l}n \\ k\end{array}\right]=\frac{[n] !}{[k ! ! n-k] !}$ if $n \geq k,\left[\begin{array}{l}n \\ k\end{array}\right]=0$ if $n<k$.

$(x-a)^{(n)}=(x-a)(x-a q) \ldots\left(x-a q^{n-1}\right)$ if $n \geq 1,(x-a)^{(0)}=1$.

$\left\{\begin{array}{l}x \\ k\end{array}\right\}=\frac{(x / a-1)(x /(a q)-1) \ldots\left(x /\left(a q^{k-1}\right)-1\right)}{\left(q^{k}-1\right) \ldots(q-1)}$ if $k \geq 1,\left\{\begin{array}{l}x \\ 0\end{array}\right\}=1$.

\section{Lemma 6.}
i) $\left[\begin{array}{l}n \\ k\end{array}\right]=\left[\begin{array}{l}n-1 \\ k-1\end{array}\right]+q^{k}\left[\begin{array}{c}n-1 \\ k\end{array}\right]$
ii) $\left[\begin{array}{l}n \\ k\end{array}\right]$ is a polynomial in $q$.
iii) $\left|\left[\begin{array}{l}n \\ k\end{array}\right]\right| \leq 1$.

Proof:

i) follows immediately from the definition, ii) and iii) follow from i). 
The polynomials $\left[\begin{array}{l}n \\ k\end{array}\right]$ are the Gauss-polynomials.

We will need the following properties of these symbols: $\left\|\left\{\begin{array}{l}x \\ k\end{array}\right\}\right\|=$ 1 , since $\left[\begin{array}{l}n \\ k\end{array}\right]=\left\{\begin{array}{l}x \\ k\end{array}\right\}$ if $x=a q^{n},\left|\left[\begin{array}{l}n \\ k\end{array}\right]\right| \leq 1$ for all $n, k$ in $\mathbb{N}$, $\left\{\begin{array}{c}a q^{k} \\ k\end{array}\right\}=\left[\begin{array}{l}k \\ k\end{array}\right]=1$ and since $\left\{\begin{array}{l}x \\ k\end{array}\right\}$ is continuous $\frac{(x-a)^{(n)}}{[n] !}=\left\{\begin{array}{l}x \\ n\end{array}\right\}$ $(q-1)^{n} q^{n(n-1) / 2} a^{n}$, so $\left\|\frac{(x-a)^{(n)}}{[n] !}\right\|=\left|(q-1)^{n}\right|$.

Definition.

If $f: V_{q} \rightarrow K$ then we define the operator $D_{q}$ as follows:

$$
\left(D_{q} f\right)(x)=\frac{f(q x)-f(x)}{x(q-1)} .
$$

The following properties are easily verified:

$$
\begin{array}{ll}
D_{q}^{j} x^{k}=[k][k-1] \ldots[k-j+1] x^{k-j} & \text { if } k \geq j \geq 1, \\
D_{q}^{j} x^{k}=0 & \text { if } k<j \\
D_{q}^{j}(x-y)^{(k)}=[k][k-1] \ldots[k-j+1](x-y)^{(k-j)} & \text { if } k \geq j \geq 1, \\
D_{q}^{j}(x-y)^{(k)}=0 & \text { if } j>k .
\end{array}
$$

\section{Lemma 7 .}

$$
x^{n}=\sum_{k=0}^{n}\left[\begin{array}{l}
n \\
k
\end{array}\right] a^{n-k}(x-a)^{(k)}
$$

Proof:

We know we can write $x^{n}$ as $x^{n}=\sum_{k=0}^{n} a_{k}(x-a)^{(k)}$. Since $D_{q} x^{n}=$ $[n] x^{n-1}$ and if we apply the operator $D_{q} k$ times and put $x=a$, we find $[n][n-1] \ldots[n-k+1] a^{n-k}=a_{k}[k] !$.

Lemma 7 and it's proof can also be found in [5, p. 121].

\section{Lemma 8.}

Take on injective sequence $\left(u_{n}\right)$ in $V_{q}$ and define

$$
q_{n}(x)=\frac{\left(x-u_{0}\right) \ldots\left(x-u_{n-1}\right)}{\left(u_{n}-u_{0}\right) \ldots\left(u_{n}-u_{n-1}\right)} \text { for } n \geq 1, \quad q_{0}(x)=1 .
$$

Then $\left(q_{n}\right)$ forms a normal basis for $C\left(V_{q} \rightarrow K\right)$ if and only if $\left\|q_{n}\right\|=1$ for all $n$.

Proof:

Put $M=V_{q}$ in Theorem 1. 


\section{Corollary.}

$\left(\left\{\begin{array}{l}x \\ n\end{array}\right\}\right)$ forms a normal basis for $C\left(V_{q} \rightarrow K\right)$.

Proof:

Put $u_{n}=a q^{n}$.

\section{Theorem 4.}

Let $\left(p_{n}(x)\right)$ be a polynomial sequence in $K[x]$ which forms a normal basis for $C\left(V_{q} \rightarrow K\right)$, and let $\left(r_{n}(x)\right)$ be a polynomial sequence such that $r_{n}(x)=\sum_{j=0}^{n} c_{n ; j} p_{j}(x)=\sum_{j=0}^{n} b_{n ; j} \mathcal{I}^{j}, c_{n: j}, b_{n ; j} \in K, c_{n: n} \neq 0, b_{n: n} \neq 0$.

Then the following are equivalent:

i) $\left(r_{n}(x)\right)$ forms a normal basis for $C\left(V_{q} \rightarrow K\right)$.

ii) $\left\|r_{n}\right\|=1,\left|c_{n ; n}\right|=1$.

iii) $\left|c_{n ; j}\right| \leq 1,\left|c_{n ; n}\right|=1$.

iv) $\left\|r_{n}\right\|=1,\left|b_{n ; n}\right|=\frac{1}{\|[n] !(q-1)^{n} \mid}$.

v) $\left|\sum_{j=k}^{n} b_{n ; j}\left[\begin{array}{l}j \\ k\end{array}\right] a^{j}\right| \leq \frac{1}{\left|[k] !(q-1)^{k}\right|},\left|b_{n ; n}\right|=\frac{1}{\mathrm{i}[n] !(q-1)^{n} \mid}$.

\section{Proof:}

i) $\Leftrightarrow$ ii) $\Leftrightarrow$ iii) follows from Theorem 3 , by putting $M=V_{q}$, ii) $\Leftrightarrow$ iv) and iii) $\Leftrightarrow$ v) follow from Lemma 7 , by putting $p_{n}(x)=\left\{\begin{array}{l}x \\ n\end{array}\right\}$.

\section{Some examples.}

1) Put $p_{n}(x)=\left\{\begin{array}{c}q x \\ n\end{array}\right\}$.

Then the sequence $\left(p_{n}(x)\right)$ forms a normal basis of $C\left(V_{q} \rightarrow K\right)$ : apply iv).

2) If the polynomial sequence $\left(p_{n}(x)\right)$ forms a normal basis of $C\left(V_{q} \rightarrow K\right)$, then so does $\left(p_{n}(q x)\right)$ : If $p_{n}(x)=\sum_{j=0}^{n} c_{n_{i j}}\left\{\begin{array}{l}x \\ j\end{array}\right\}$, then $p_{n}(q x)=\sum_{j=0}^{n} c_{n ; j}\left\{\begin{array}{c}q x \\ j\end{array}\right\}$.

Use cxample i) and apply iii).

3) If the sequence $\left(p_{n}(x)\right)$ forms a normal basis of $C\left(V_{q} \rightarrow K\right)$, then so does $\left(p_{n}\left(q^{k} x\right)\right)$ where $k$ is a fixed natural number: use Example 2). 
4) If the sequence $\left(p_{n}(x)\right)$ forms a normal basis of $C\left(V_{q} \rightarrow K\right)$, then so does $\left(p_{n}\left(q^{k_{n}} x\right)\right)$, where $\left(k_{n}\right)$ is a sequence in $\mathbb{N}$ : usc Example 3).

5) If the sequence $\left(p_{n}(x)\right)$ forms a normal basis of $C\left(V_{q} \rightarrow K\right)$, then so does $\left(r_{n}(x)\right)$, where $r_{n}(x)=p_{0}(x)+p_{1}(x)+\cdots+p_{n}(x)$ : apply iii).

6) If the polynomial sequence $\left(p_{n}(x)\right)$ forms a normal basis of $C\left(V_{q} \rightarrow K\right)$, then so does $\left((q-1)^{j} D_{q}^{j} p_{n}(x)\right)_{n \geq j}, j \in \mathbb{N}, j$ fixed: apply iii).

To end this chapter, we give the valuation of $\left|b_{n ; n}\right|=\frac{1}{\left|[n] !(q-1)^{n}\right|}$.

If $n$ is different from zero, then $\frac{1}{\left|[n] !(q-1)^{n}\right|}=\frac{1}{\left|\left(q^{n}-1\right)\left(q^{n-1}-1\right) \ldots(q-1)\right|}$ and this leads us to the following lemma:

\section{Lemma 9.}

Take $m \geq 1, m$ the smallest integer such that $q^{m} \equiv 1(\bmod p)$.

i) If $q^{m} \equiv 1\left(\bmod p^{r}\right), q^{m} \not \equiv 1\left(\bmod p^{r+1}\right)(r \geq 1)$, and $(p, r) \neq(2,1)$ then

$$
\left|\left(q^{k}-1\right)\left(q^{k-1}-1\right) \ldots(q-1)\right|=p^{-[k / m] r}|[k / m] !|
$$

where $[x]=\max \{k \in \mathbb{Z} \mid k \leq x\}$.

ii) If $q \equiv 3(\bmod 4)$, where

$$
\begin{aligned}
& q=1+2+2^{2} \varepsilon_{,} \\
& \varepsilon=\varepsilon_{0}+\varepsilon_{1} 2+\varepsilon_{2} 2^{2}+\ldots, \varepsilon_{0}=\varepsilon_{1}=\cdots=\varepsilon_{N-1}=1, \varepsilon_{N}=0
\end{aligned}
$$

then

$$
\begin{aligned}
& \left|\left(q^{k}-1\right)\left(q^{k-1}-1\right) \ldots(q-1)\right| \\
& =2^{-2 k} 2^{-N k / 2}|(k / 2) !| \\
& =2^{(-k N-4 k+N+2) / 2} 2^{-N k / 2}|((k-1) / 2) \ddagger| \text { if } k \text { is odd. }
\end{aligned}
$$

We remark that (see [4, Section 25.5]) $|j \ddagger|=p^{-\lambda(j)}$ with $\lambda(j)=\frac{j-s_{j}}{p-1}$,

$$
j=\sum_{i=0}^{t} \gamma_{i} p^{i}, \quad s_{j}=\sum_{i=0}^{t} \gamma_{i} .
$$

Proof:

i) Suppose $q^{m} \equiv 1\left(\bmod p^{r}\right), q^{m} \neq 1\left(\bmod p^{r+1}\right), r \geq 1,(p, r) \neq(2,1)$. First, take $p \neq 2 \cdot q^{k}-1=q^{m j+s}-1$ with $0 \leq s<m$. 
Then $\left(q^{m}\right)^{j} \equiv 1\left(\bmod p^{r+\operatorname{ord}_{p} j}\right),\left(q^{m}\right)^{j} \not \equiv 1\left(\bmod p^{r+1+\operatorname{ord}_{p} j}\right)$ (Lemma 2), so $\left(q^{m}\right)^{j}=1+\alpha p^{\tau+\operatorname{ord}_{p} j} \operatorname{ord}_{p} \alpha=0$.

If $s$ is different from zero, then $q^{s}=\beta$ with $\beta=\beta_{0}+\beta_{1} p+\beta_{2} p^{2}+\ldots$ with $\beta_{0} \neq 0, \beta_{0} \neq 1$, so $q^{m j+s}-1=\left(1+\alpha p^{r+\operatorname{ord}_{p} j}\right) \beta-1=\beta+$ $\alpha \beta p^{r+o r d_{p} j}-1$ and thus $q^{m j+s}-1$ is a unit if $s$ is different from zero.

Then

$$
\begin{aligned}
\left|\left(q^{k}-1\right)\left(q^{k-1}-1\right) \ldots(q-1)\right| & =\left|\left(\left(q^{7 n}\right)^{j}-1\right) \ldots\left(q^{m}-1\right)\right| \\
& =p^{-\left(r+\operatorname{ord}_{p} j\right)} \ldots p^{-\left(r+\operatorname{ord}_{p} 1\right)} \\
& \left.=p^{-r j}|j !|=p^{-[k / m] r}|| k / m\right] ! \mid
\end{aligned}
$$

If $p$ is equal to 2 then $m$ equals one and thus

$$
\begin{aligned}
\left|\left(q^{k}-1\right)\left(q^{k-1}-1\right) \ldots(q-1)\right| & =2^{-\left(r+o r d_{p} k\right)} \ldots 2^{-\left(r+\operatorname{ord}_{p} 1\right)} \\
& =2^{-r k}|k !|=2^{-[k / m) r} \mid[k / m] ! !
\end{aligned}
$$

ii) Suppose $q \equiv 3(\bmod 4)$. We use Lemma 3 .

If $k$ is even then $\left|\left(q^{k}-1\right)\left(q^{k-1}-1\right) \ldots(q-1)\right|$

$$
\begin{aligned}
& =2^{-k / 2} 2^{-\left(N+2+\operatorname{ord}_{2} k\right)} 2^{-\left(N+2+\operatorname{ord}_{2}(k-2)\right)} \ldots 2^{-\left(N+2+\operatorname{ord}_{2} 2\right)} \\
& =2^{-k / 2} 2^{-(N+2) k / 2}|k||k-2| \ldots|2| \\
& =2^{-2 k} 2^{-N k / 2}|(k / 2) \ddagger|
\end{aligned}
$$

and if $k$ is odd $\left|\left(q^{k}-1\right)\left(q^{k-1}-1\right) \ldots(q-1)\right|$

$$
\begin{aligned}
& =2^{-(k+1) / 2} 2^{-\left(N+2+\operatorname{ord}_{2}(k-1)\right)} 2^{-\left(N+2+\text { ord }_{2}(k-3)\right)} \ldots 2^{-\left(N+2+\text { ord }_{2} 2\right)} \\
& =2^{-(k+1) / 2} 2^{-(N+2)(k-1) / 2} 2^{-(k-1) / 2}|((k-1) / 2) !| \\
& =2^{(-N k-4 k+N+2) / 2}|((k-1) / 2) !| \text { which proves the lemma. }
\end{aligned}
$$

\section{More bases for $C\left(V_{q} \rightarrow \mathbb{Q}_{p}\right)$}

We want to make new normal bases, using the basis $\left(\left\{\begin{array}{l}x \\ n\end{array}\right\}\right)$.

Now, if $E$ is a non-archimedean Banach space over a non-archimcdean valued field $L$, and $E$ has a normal basis, then the norm of $E$ satisfies the following condition: for each element $x$ of $E$ thcre exists $\nu$ in $L$ such that the norm of $x$ is equal to $|\nu|$. Y. Amice $([2$, p. 82]) calls this condition $(N)$. 
So, if we want to make more normal bases for $C\left(V_{q} \rightarrow \mathbb{Q}_{p}\right)$ we can use the following result $([2$, p. 82 , Prop. 3.1 .5$])$ :

Let $E$ be a Banach space over a non-archimedean valued field $L$. If $L$ has a discrete valuation and if $E$ satisfies condition $(N)$, then for a family $\left(e_{n}\right)$ of $E$ for which $\left\|e_{n}\right\| \leq 1$ for all $n$ the following are equivalent:

i) $\left(e_{n}\right)$ is a normal basis of $E$,

ii) $\left(p\left(e_{n}\right)\right)$ is a basis of the vector space $\bar{E}$.

where $E_{0}=\{x \in E \mid\|e\| \leq 1\}, E_{0}^{\prime}=\{x \in E \mid\|e\|<1\}, \bar{E}=E_{0} / E_{0}^{\prime}$ and $p$ is the canonical projection of $E_{0}$ on $\bar{E}$.

Since the valuation of $L$ has to be discrete, we use this result to find normal bases for $C\left(V_{q} \rightarrow \mathbb{Q}_{p}\right)$. We start with some lemmas.

Lermma 10.

$$
\left[\begin{array}{c}
i+j \\
n
\end{array}\right]=\sum_{k=0}^{n}\left[\begin{array}{l}
i \\
k
\end{array}\right]\left[\begin{array}{c}
j \\
n-k
\end{array}\right] q^{-k(-j+n-k)}
$$

Proof:

If $n$ is zero or $i+j$ is strictly smaller then $n$, then the lemma surely holds.

From now on we suppose $i+j$ greater than $n$.

If $i+j$ is equal to $n$ then $\left[\begin{array}{c}i+j \\ n\end{array}\right]$ is equal to one and $\sum_{k=0}^{n}\left[\begin{array}{l}i \\ k\end{array}\right]\left[\begin{array}{c}j \\ n-k\end{array}\right] q^{-k(-j+n-k)}=\sum_{k=0}^{n}\left[\begin{array}{l}i \\ k\end{array}\right]\left[\begin{array}{c}n-i \\ n-k\end{array}\right] q^{-k(i-k)}=1$ since the only term different from zero is the term where $k$ equals $i$.

From now on we proceed by (double) induction.

$$
\begin{aligned}
{\left[\begin{array}{c}
i+j \\
n
\end{array}\right]=} & {\left.\left[\begin{array}{c}
i+j-1 \\
n-1
\end{array}\right]+q^{n}\left[\begin{array}{c}
i+j-1 \\
n
\end{array}\right] \text { (by Lemma } 6\right) } \\
= & \sum_{k=0}^{n-1}\left[\begin{array}{l}
i \\
k
\end{array}\right]\left[\begin{array}{c}
j-1 \\
n-k-1
\end{array}\right] q^{-k(-j+n-k)} \\
& +q^{n} \sum_{k=0}^{n}\left[\begin{array}{l}
i \\
k
\end{array}\right]\left[\begin{array}{c}
j-1 \\
n-k
\end{array}\right] q^{-k(-j+1+n-k)} \\
= & {\left[\begin{array}{c}
i \\
n
\end{array}\right] q^{n j}+\sum_{k=0}^{n-1}\left[\begin{array}{c}
i \\
k
\end{array}\right] q^{-k(-j+n-k)}\left(\left[\begin{array}{c}
j-1 \\
n-k-1
\end{array}\right]+\left[\begin{array}{l}
j-1 \\
n-k
\end{array}\right] q^{n-k}\right) } \\
= & \sum_{k=0}^{n}\left[\begin{array}{l}
i \\
k
\end{array}\right]\left[\begin{array}{c}
j \\
n-k
\end{array}\right] q^{-k(-j+n-k)} \text { (by Lemma 6). }
\end{aligned}
$$




\section{Lemma 11.}

Let $q^{m} \equiv 1\left(\bmod p^{k_{0}}\right), q^{m} \not \equiv 1\left(\bmod p^{k_{0}+1}\right)$ with $\left(p, k_{0}\right) \neq(2,1)$.

If $x, y \in V_{q},|x-y| \leq p^{-\left(k_{0}+t\right)}$ then $\left|\left\{\begin{array}{l}x \\ n\end{array}\right\}^{s}-\left\{\begin{array}{c}y \\ n\end{array}\right\}^{s}\right| \leq 1 / p$, where $s \in \mathbb{N}, 0 \leq n<m p^{t}$.

\section{Proof:}

The lemma holds if $s$ is equal to zero.

If $q^{m} \equiv 1\left(\bmod p^{k_{0}}\right), q^{m} \not \equiv 1\left(\bmod p^{k_{0}+1}\right)$ with $\left(p, k_{0}\right) \neq(2,1)$, we then have $V_{q}=\underset{0 \leq r \leq m-1}{\bigcup}\left\{x \in \mathbb{Z}_{p}|| x-a q^{r} \mid \leq p^{-k_{0}}\right\}$ (Lemma 4).

So $V_{q}$ is the union of $m$ disjoint balls with radius $p^{-k_{0}}$.

By the proof of Lemma 4 , we have that $V_{q}$ is the union of $m p^{t}$ disjoint balls with radius $p^{-\left(k_{0}+t\right)}$ and with centers $a q^{r}\left(q^{m}\right)^{k}, 0 \leq r \leq m-1$, $0 \leq k<p^{t}$.

Take $x, y \in\left\{a q^{j} \mid j=0,1,2, \ldots\right\}$ with $|x-y| \leq p^{-\left(k_{0}+t\right)}$. Then, by Lemmas 2 and 4 , there exist natural numbers $n_{x}$ and $n_{y}$ such that $x=a q^{r}\left(q^{m}\right)^{n_{x}}$ and $y=a q^{r}\left(q^{m}\right)^{n_{y}}$ with $\left|n_{x}-n_{y}\right| \leq p^{-t}\left(n_{x}, n_{y} \in \mathbb{N}\right)$.

Then

$$
\left|\left\{\begin{array}{l}
x \\
n
\end{array}\right\}-\left\{\begin{array}{l}
y \\
n
\end{array}\right\}\right|=\left|\left[\begin{array}{c}
r+m n_{x} \\
n
\end{array}\right]-\left[\begin{array}{c}
r+m n_{y} \\
n
\end{array}\right]\right| .
$$

Further,

$$
\begin{aligned}
{\left[\begin{array}{c}
r+m n_{x} \\
n
\end{array}\right] } & =\left[\begin{array}{c}
m\left(n_{x}-n_{y}\right)+r+m n_{y} \\
n
\end{array}\right] \\
& =\sum_{k=0}^{n}\left[\begin{array}{c}
m\left(n_{x}-n_{y}\right) \\
k
\end{array}\right]\left[\begin{array}{c}
r+m n_{y} \\
n-k
\end{array}\right] q^{-k\left(-\left(r+m n_{y}\right)+n-k\right)}
\end{aligned}
$$

(Lemma 10 if $n_{x} \geq n_{y}$ )

$$
\begin{aligned}
= & {\left[\begin{array}{c}
r+m n_{y} \\
n
\end{array}\right] } \\
& +\sum_{k=1}^{n}\left[\begin{array}{c}
m\left(n_{x}-n_{y}\right) \\
k
\end{array}\right]\left[\begin{array}{c}
r+m n_{y} \\
n-k
\end{array}\right] q^{-k\left(-\left(r+m n_{y}\right)+n-k\right)}
\end{aligned}
$$

$$
(n \geq 1)
$$

Since $\left[\begin{array}{l}i \\ j\end{array}\right]=\frac{[i]}{[j]}\left[\begin{array}{l}i-1 \\ j-1\end{array}\right](i \geq j \geq 1)$ we have $\left|\left[\begin{array}{l}i \\ j\end{array}\right]\right| \leq\left|\frac{[i]}{[j]}\right|(i \geq 0, j \geq 1)$, so $\left|\left[\begin{array}{c}m\left(n_{x}-n_{y}\right) \\ k\end{array}\right]\right| \leq\left|\frac{\left[m\left(n_{x}-n_{y}\right)\right]}{[k]}\right|=\left|\frac{\left(q^{m}\right)^{n_{x}-n_{y}}-1}{q^{k}-1}\right| \leq 1 / p$ by Lemma 2 since $1 \leq k \leq n<m p^{t}$ and $\left|n_{x}-n_{y}\right| \leq p^{-t}$. 
Then

$$
\begin{aligned}
& \left|\left[\begin{array}{c}
r+m n_{x} \\
n
\end{array}\right]-\left[\begin{array}{c}
r+m n_{y} \\
n
\end{array}\right]\right| \\
& \leq \max _{1 \leq k \leq n}\left\{\left|\left[\begin{array}{c}
m\left(n_{x}-n_{y}\right) \\
k
\end{array}\right]\left[\begin{array}{c}
r+m n_{y} \\
n-k
\end{array}\right] q^{-k\left(-\left(r+m_{y}\right)+n-k\right)}\right|\right\} \leq 1 / p .
\end{aligned}
$$

So $\left\{\left\{\begin{array}{l}x \\ n\end{array}\right\}-\left\{\begin{array}{l}y \\ n\end{array}\right\} \mid \leq 1 / p\right.$ and this also holds if $n$ is zcro.

Finally, if $s$ is greater then one,

$$
\left|\left\{\begin{array}{l}
x \\
n
\end{array}\right\}^{s}-\left\{\begin{array}{l}
y \\
n
\end{array}\right\}^{s}\right|=\left|\left\{\begin{array}{l}
x \\
n
\end{array}\right\}-\left\{\begin{array}{l}
y \\
n
\end{array}\right\}\right|\left|\sum_{i=0}^{s-1}\left\{\begin{array}{l}
x \\
n
\end{array}\right\}^{i}\left\{\begin{array}{l}
y \\
n
\end{array}\right\}^{s-1-i}\right| \leq 1 / p
$$

The lemma follows by continuity.

\section{Lemma 12.}

Let $q \equiv 3(\bmod 4)$,

$$
\begin{aligned}
& q=1+2+2^{2} \varepsilon \\
& \varepsilon=\varepsilon_{0}+\varepsilon_{1} 2+\varepsilon_{2} 2^{2}+\ldots, \varepsilon_{0}=\varepsilon_{1}=\cdots=\varepsilon_{N-1}=1, \varepsilon_{N}=0 .
\end{aligned}
$$

If $x, y \in V_{q},|x-y| \leq p^{-(N+2+t)}$ then $\left|\left\{\begin{array}{l}x \\ n\end{array}\right\}^{s}-\left\{\begin{array}{l}y \\ n\end{array}\right\}^{s}\right| \leq 1 / 2$, where $s \in \mathbb{N}, 0 \leq n<2^{t}(t \geq 1)$.

Proof:

The lemma holds if $s$ is equal to zcro.

$V_{q}=\left\{x \in \mathbb{Z}_{2}|| x-a \mid \leq 2^{-(N+3)}\right\} \cup\left\{x \in \mathbb{Z}_{2}|| x-a q \mid \leq 2^{-(N+3)}\right\}$, by Lemma 5 .

By the proof of Lemma 5, we have that $V_{q}$ is the union of $2^{t}$ disjoint balls with radius $2^{-(N+2+t)}$ and with centers $a q^{n}: 0 \leq n<2^{t}(t \geq 1)$.

Take $x, y \in\left\{a \dot{q}^{j} \mid j=0,1,2, \ldots\right\}$ with $|x-y| \leq 2^{-(N+2+t)}$. Then. by Lemmas 3 and 5 we must have that $x=a q^{n_{x}}$ and $y=a q^{n_{y}}$ with $\left|n_{x}-n_{y}\right| \leq 2^{-t}\left(n_{x}, n_{y} \in \mathbb{N}\right)$. Then

$$
\left|\left\{\begin{array}{l}
x \\
n
\end{array}\right\}-\left\{\begin{array}{l}
y \\
n
\end{array}\right\}\right|=\left|\left[\begin{array}{c}
n_{x} \\
n
\end{array}\right]-\left[\begin{array}{c}
n_{y} \\
n
\end{array}\right]\right| .
$$


Further,

$$
\begin{aligned}
{\left[\begin{array}{c}
n_{x} \\
n
\end{array}\right] } & =\left[\begin{array}{c}
\left(n_{x}-n_{y}\right)+n_{y} \\
n
\end{array}\right] \\
& \left.=\sum_{k=0}^{n}\left[\begin{array}{c}
n_{x}-n_{y} \\
k
\end{array}\right]\left[\begin{array}{c}
n_{y} \\
n-k
\end{array}\right] q^{-k\left(-n_{y}+n-k\right)} \text { (Lemma, } 10 \text { if } n_{x} \geq n_{y}\right) \\
& =\left[\begin{array}{c}
n_{y} \\
n
\end{array}\right]+\sum_{k=1}^{n}\left[\begin{array}{c}
n_{x}-n_{y} \\
k
\end{array}\right]\left[\begin{array}{c}
n_{y} \\
n-k
\end{array}\right] q^{-k\left(-n_{y}+n^{-k)}\right.}(n \geq 1)
\end{aligned}
$$

Since $\left[\begin{array}{l}i \\ j\end{array}\right]=\frac{i j]}{[j]}\left[\begin{array}{l}i-1 \\ j-1\end{array}\right](i \geq j \geq 1)$ we have $\left|\left[\begin{array}{l}i \\ j\end{array}\right]\right| \leq\left|\frac{[i]}{[j]}\right|(i \geq 0, j \geq 1)$, so $\left|\left[\begin{array}{c}n_{x}-n_{y} \\ k\end{array}\right]\right| \leq\left|\frac{\left[n_{x}-n_{y}\right]}{[k]}\right|=\left|\frac{a^{n_{x}-n_{y}-1}}{q^{k}-1}\right| \leq 1 / 2$ by Lemma 3 since $1 \leq k \leq n<2^{t}$ and $\left|n_{x}-n_{y}\right| \leq 2^{-t}$.

Then

$\left|\left[\begin{array}{c}n_{x} \\ n\end{array}\right]-\left[\begin{array}{c}n_{y} \\ n\end{array}\right]\right| \leq \max _{1 \leq k \leq n}\left\{\left|\left[\begin{array}{c}n_{x}-n_{y} \\ k\end{array}\right]\left[\begin{array}{c}n_{y} \\ n-k\end{array}\right] q^{-k\left(-n_{y}+n-k\right)}\right|\right\} \leq 1 / 2$.

So $\left\{\left\{\begin{array}{l}x \\ n\end{array}\right\}-\left\{\begin{array}{l}y \\ n\end{array}\right\} \mid \leq 1 / 2\right.$ and this also holds if $n$ is zero.

Finally, if $s$ is greater then one,

$$
\left|\left\{\begin{array}{l}
x \\
n
\end{array}\right\}^{5}-\left\{\begin{array}{l}
y \\
n
\end{array}\right\}^{s}\right|=\left|\left\{\begin{array}{l}
x \\
n
\end{array}\right\}-\left\{\begin{array}{l}
y \\
n
\end{array}\right\}\right|\left|\sum_{i=0}^{s-1}\left\{\begin{array}{l}
x \\
n
\end{array}\right\}^{i}\left\{\begin{array}{l}
y \\
n
\end{array}\right\}^{s-1-i}\right| \leq 1 / 2 .
$$

The lemma follows by continuity.

Since $C\left(V_{q} \rightarrow \mathbb{Q}_{p}\right)$ has a normal basis, its norm satisfies condition $(N)$, and so we can use $[2$, p. 82 , Prop. 3.1 .5$]$ to prove the following:

Theorem 5.

Let $\left(j_{n}\right)$ be a sequence in $\mathbb{N}_{0}$. Then the sequence of polynomials $\left(\left\{\begin{array}{l}x \\ n\end{array}\right\}^{j_{n}}\right)$ forms a normal basis for $C\left(V_{q} \rightarrow \mathbb{Q}_{p}\right)$.

\section{Proof:}

This proof is analogous to the proof of Theorem $1.1 \mathrm{in}[3]$.

By [2, Proposition 3.1.5, p. 82] it suffices to prove that $\left(\left\{\begin{array}{l}x \\ n\end{array}\right\}^{j_{n}}\right)$ forms a vectorial basis of $C\left(V_{q} \rightarrow \mathbb{F}_{p}\right)$. 
We distinguish two cases.

If $q^{m} \equiv 1\left(\bmod p^{k_{0}}\right), q^{m} \neq 1\left(\bmod p^{k_{0}+1}\right)$ with $\left(p, k_{0}\right) \neq(2,1)$, define $C_{t}$ the space of the functions from $V_{q}$ to $\mathbb{F}_{p}$ constant on balls of the type $\left\{x \in \mathbb{Z}_{p}|| x-\alpha \mid \leq p^{-\left(k_{0}+t\right)}\right\}, \alpha \in V_{q}$. Since $C\left(V_{q} \rightarrow \mathbb{F}_{p}\right)=\cup_{t \geq 0} C_{t}$ it suffices to prove that $\left(\overline{\left\{\begin{array}{l}x \\ n\end{array}\right\}^{j_{n}}} \mid n<m p^{t}\right)$ forms a basis of $C_{t}$. By the proof of Lemma 4, we can write $V_{q}$ as the union of $m p^{t}$ disjoint balls with radius $p^{-\left(k_{0}+t\right)}$ and with centers $a q^{r}\left(q^{m}\right)^{n}, 0 \leq r \leq m-1,0 \leq n<p^{t}$. Let $\chi_{i}$ be the charactcristic function of the ball with center $a q^{i}$. Using Lemma 11, we have

$$
\overline{\left\{\begin{array}{l}
x \\
n
\end{array}\right\}^{j_{n}}}=\sum_{i=0}^{m p^{t}-1} \chi_{i}(x) \overline{\left\{\begin{array}{c}
a q^{i} \\
n
\end{array}\right\}^{j_{n}}}=\sum_{i=n}^{m p^{t}-1} \chi_{i}(x) \overline{\left\{\begin{array}{c}
a q^{i} \\
n
\end{array}\right\}^{j_{n}}},
$$

hence the transition matrix from $\left(\chi_{n} \mid n<m p^{t}\right)$ to $\left(\overline{\left\{\begin{array}{l}x \\ n\end{array}\right\}^{j_{n}}} \mid n<m p^{t}\right)$ is triangular, so $\left(\overline{\left\{\begin{array}{l}x \\ n\end{array}\right\}^{j_{n}}} \mid n<m p^{t}\right)$ forms a basis of $C_{t}$.

If $q \equiv 3(\bmod 4), q=1+2+2^{2} \varepsilon, \varepsilon=\varepsilon_{0}+\varepsilon_{1} 2+\varepsilon_{2} 2^{2}+\ldots, \varepsilon_{0}=\varepsilon_{1}=$ $\cdots=\varepsilon_{N-1}=1, \varepsilon_{N}=0$, define $C_{t}$ the space of the functions from $V_{q}$ to $\mathbb{F}_{2}$ constant on balls of the type $\left\{x \in \mathbb{Z}_{2}|| x-\alpha \mid \leq 2^{-(N+2+t)}\right\}, \alpha \in V_{q}$.

Since $C\left(V_{q} \rightarrow \mathbb{F}_{2}\right)=\cup_{t \geq 1} C_{t}$ it suffices to prove that $\left(\overline{\left\{\begin{array}{l}x \\ n\end{array}\right\}^{j_{n}} \mid n<2^{t}}\right)$ forms a basis of $C_{t}$. By the proof of Lemma 5 , we can write $V_{q}$ as the union of $2^{t}$ disjoint balls with radius $2^{-(N+2+t)}$ and with centers $a q^{n}$, $0 \leq n<2^{t}$. Let $\chi_{i}$ be the characteristic function of the ball with center $a q^{i}$. Using Lemma 12 we have

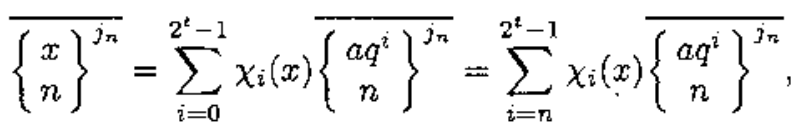

hence the transition matrix from $\left(\chi_{n} \mid n<2^{t}\right)$ to $\left(\overline{\left\{\begin{array}{l}x \\ n\end{array}\right\}^{j_{n}}} \mid n<2^{t}\right)$ is triangular, so $\left(\overline{\left\{\begin{array}{l}x \\ n\end{array}\right\}^{j_{n}}} \mid n<2^{t}\right)$ forms a basis of $C_{t}$. This proves the theorem. 


\section{Extension to $C\left(V_{q} \rightarrow K\right)$}

Let $K$ be as in Chapters 3 and 4 . We want to show that $\left(\left\{\begin{array}{l}x \\ n\end{array}\right\}^{j_{n}}\right)$ forms a normal basis for $C\left(V_{q} \rightarrow K\right)$. To prove this, we need the results from Scction 5. We remark that the valuation of $K$ does not have to be discrete, as was the case in Section 5.

\section{Theorem 6.}

Let $\left(j_{n}\right)$ be a sequence in $\mathbb{N}_{0}$. Then $\left(\left\{\begin{array}{l}x \\ n\end{array}\right\}^{j_{n}}\right)$ forms a normal basis for $C\left(V_{q} \rightarrow K\right)$.

Proof:

It is clear that $\left\|\left\{\begin{array}{l}x \\ n\end{array}\right\}^{j_{n}}\right\|=1$.

We now prove the orthogonality of the sequence. Let $n$ be in $\mathbb{N}, \alpha_{0}, \ldots, \alpha_{n}$ in $K$. We prove $\left\|\alpha_{0}\left\{\begin{array}{l}x \\ 0\end{array}\right\}^{j_{0}}+\cdots+\alpha_{n}\left\{\begin{array}{l}x \\ n\end{array}\right\}^{j_{n}}\right\|=$ $\max _{0 \leq i \leq n}\left\{\left|\alpha_{i}\right|\right\}$.

It is clear that $\left\|\alpha_{0}\left\{\begin{array}{l}x \\ 0\end{array}\right\}^{j_{0}}+\cdots+\alpha_{n}\left\{\begin{array}{l}x \\ n\end{array}\right\}^{j_{n}}\right\| \leq \max _{0 \leq i \leq n}\left\{\left|\alpha_{i}\right|\right\}$.

Put $M=\max _{0 \leq i \leq n}\left\{\left|\alpha_{i}\right|\right\}, N=\min \left\{i \mid 0 \leq i \leq n\right.$ and $\left.\left|\alpha_{i}\right|=M\right\}$. Then

$\| \alpha_{0}\left\{\begin{array}{c}a q^{N} \\ 0\end{array}\right\}^{j_{0}}+\cdots+\alpha_{n}\left\{\begin{array}{c}a q^{N} \\ n\end{array}\right\}^{j_{n}}$

$=\max \left\{\left|\alpha_{0}\left\{a q^{N}\right\}^{j_{0}}+\cdots+\alpha_{N-1}\left\{\begin{array}{c}a q^{N} \\ N-1\end{array}\right\}^{j_{N-1}}\right|,\left|\alpha_{N}\left\{a q^{N}\right\}^{j_{N}}\right|\right\}$

$=\left|\alpha_{N}\left\{\begin{array}{c}a q^{N} \\ N\end{array}\right\}^{j_{N}}\right|=M$

since $\left|\alpha_{0}\left\{\begin{array}{c}a q^{N} \\ 0\end{array}\right\}^{j_{0}}+\cdots+\alpha_{N-1}\left\{\begin{array}{c}a q^{N} \\ N-1\end{array}\right\}^{j_{N-1}}\right|<M,\left|\alpha_{N}\left\{\begin{array}{c}a q^{N} \\ N\end{array}\right\}^{j_{N}}\right|=$ $M$. So $\left\|\alpha_{0}\left\{\begin{array}{l}x \\ 0\end{array}\right\}^{j_{0}}+\cdots+\alpha_{n}\left\{\begin{array}{l}x \\ n\end{array}\right\}^{j_{n}}\right\|=\max _{0 \leq i \leq n}\left\{\left|\alpha_{i}\right|\right\}$.

Finally, we prove that the sequence forms a basis. 
By $[6$, p. 165, Lemma 5.1] and by Kaplansky's Theorem (see e.g. $[6$, p. 191, Theorem 5.28]), it suffices to prove that the $k$ linear span of the polynornials $\left(\left\{\begin{array}{l}x \\ n\end{array}\right\}^{j_{n}}\right)$ contains $K[x]$. Since each $\left\{\begin{array}{l}x \\ k\end{array}\right\}$ is an element of $\mathbb{Q}_{p}[x]$ and $\left(\left\{\begin{array}{l}x \\ n\end{array}\right\}^{j_{n}}\right)$ forms a normal basis of $C\left(V_{q} \rightarrow \mathbb{Q}_{p}\right)$ (Theorem 5), we can write $\left\{\begin{array}{l}x \\ k\end{array}\right\}$ as a uniformly convergent expansion $\left\{\begin{array}{l}x \\ k\end{array}\right\}=\sum_{n=0}^{\infty} a_{n} q_{n}(x)^{j_{n}}$. So if $\alpha_{0}, \alpha_{1}, \ldots, \alpha_{n}$ are elements of $K$ then there exists coefficients $d_{n}^{\left(j_{n}\right)}$ in $K$ such that $\sum_{i=0}^{n_{n}} \alpha_{i}\left\{\begin{array}{c}x \\ i\end{array}\right\}=\sum_{n=0}^{\infty} d_{n}^{\left(j_{n}\right)}\left\{\begin{array}{l}x \\ n\end{array}\right\}^{j_{n}}$ where the right-hand-side is uniformly convergent.

Let $p$ be an element of $K[x]$. By the previous remark there exist coefficients $c_{n}^{\left(j_{n}\right)}$ such that $p(x)=\sum_{i=0}^{N} \beta_{i}\left\{\begin{array}{l}x \\ n\end{array}\right\}=\sum_{n=0}^{\infty} c_{n}^{\left(j_{n}\right)}\left\{\begin{array}{l}x \\ n\end{array}\right\}^{j_{n}}$. So the $k$ linear span of the polynomials $\left(\left\{\begin{array}{l}x \\ n\end{array}\right\}^{j_{n}}\right)$ contains $K[x]$. This finishes the proof.

If $f$ is an element of $C\left(V_{q} \rightarrow K\right)$, there exist coefficients $\left(b_{n}^{\left(j_{n}\right)}\right)$ such that $f(x)=\sum_{n=0}^{\infty} b_{n}^{\left(j_{n}\right)}\left\{\begin{array}{l}x \\ n\end{array}\right\}^{j_{n}}$ where the series on the right-hand-side is uniformly convergent. In some cases it is possible to give an expression for the coefficients:

\section{Proposition 1.}

Let $s$ be in $\mathbb{N}_{0}$. Then each continuous function $f: V_{q} \rightarrow K$ can be written as a uniformly convergent series

$$
f(x)=\sum_{n=0}^{\infty} b_{n}^{(s)}\left\{\begin{array}{l}
x \\
n
\end{array}\right\} \text { with }\|f\|=\max _{n \geq 0}\left\{\left|b_{n}^{(s)}\right|\right\}
$$

where

$$
b_{n}^{(s)}=\sum_{k=0}^{n}(-1)^{n-k}\left[\begin{array}{l}
n \\
k
\end{array}\right]^{s} f\left(a q^{k}\right) \beta_{n-k}^{(s)}
$$

and $\beta_{0}^{(s)}=1, \beta_{m}^{(s)}=\sum_{\substack{\left(j_{1}, \ldots, j_{r}\right) \\ \sum j_{i}=m_{i} 1 \leq j_{i} \leq m}}(-1)^{r+m}\left[\begin{array}{c}m \\ j_{1} \ldots j_{r}\end{array}\right]^{s},\left[\begin{array}{c}m \\ j_{1} \ldots j_{r}\end{array}\right]=$ $\frac{[m] !}{\left[j_{1}\right] ! \ldots\left\lfloor\left(j_{r}\right] !\right.}$. 


\section{Proof:}

The proof is equal to the proof of Corollary $1.2 \mathrm{in}[3]$.

$\|f\|=\max _{n \geq 0}\left\{\left|b_{n}^{(s)}\right|\right\}$ follows from the fact that $\left(\left\{\begin{array}{l}x \\ n\end{array}\right\}^{s}\right)$ forms a normal basis. If $f(x)=\sum_{n=0}^{\infty} b_{n}^{(s)}\left\{\begin{array}{l}x \\ n\end{array}\right\}^{s}$ then $f\left(a q^{k}\right)=\sum_{n=0}^{k} b_{n}^{(s)}\left[\begin{array}{l}k \\ n\end{array}\right]^{s}$, and so $b_{0}^{(s)}=f(a), b_{k}^{(s)}=f\left(a q^{k}\right)-\sum_{n=0}^{k-1} b_{n}^{(s)}\left[\begin{array}{l}k \\ n\end{array}\right]^{s}$ if $k \geq 1$.

If $k$ is equal to zero, the formulas certainly hold.

We proceed by induction. Suppose the formulas hold for $0 \leq j \leq N$.

$$
\begin{aligned}
b_{N+1}^{(s)} & =f\left(a q^{N+1}\right)-\sum_{n=0}^{N} b_{n}^{(s)}\left[\begin{array}{c}
N+1 \\
n
\end{array}\right]^{s} \\
& =f\left(a q^{N+1}\right)-\sum_{n=0}^{N}\left[\begin{array}{c}
N+1 \\
n
\end{array}\right]^{s} \sum_{k=0}^{n}\left[\begin{array}{c}
n \\
k
\end{array}\right]^{s}(-1)^{n-k} f\left(a q^{k}\right) \beta_{n-k}^{(s)} \\
& =f\left(a q^{N+1}\right)-\sum_{k=0}^{N} \sum_{n=k}^{N}(-1)^{n-k} f\left(a q^{k}\right) \\
& =f\left(a q^{N+1}\right)+\sum_{k=0}^{N} \sum_{n=k}^{N} f\left(a q^{k}\right) \\
& \sum_{\sum_{i=1}^{r}}^{r}(-1)^{r+n-k}\left[\begin{array}{c}
n-k \\
j_{i}=n-k
\end{array}\right]^{s}\left[\begin{array}{c}
n \\
k
\end{array}\right]^{s}\left[\begin{array}{c}
N+1 \\
n
\end{array}\right]^{s} \\
& (-1)^{r+1}\left(\frac{[N+1] !}{\left[j_{1}\right] ! \ldots\left[j_{r}\right] ![k] ![N+1-n] !}\right)^{s}
\end{aligned}
$$

put $j_{r+1}=N+1-n$

$$
\begin{aligned}
& =f\left(a q^{N+1}\right)+\sum_{k=0}^{N}\left[\begin{array}{c}
N+1 \\
k
\end{array}\right]^{s} f\left(a q^{k}\right) \\
& \sum_{\sum_{2=1}^{r+1} j_{r}=N+1-k}(-1)^{r+1}\left[\begin{array}{c}
N+1-k \\
j_{1} \ldots j_{r+1}
\end{array}\right]^{s} \\
& =f\left(a q^{N+1}\right)+\sum_{k=0}^{N}\left[\begin{array}{c}
N+1 \\
k
\end{array}\right]^{s} f\left(a q^{k}\right)(-1)^{N+1-k} \beta_{N+1-k}^{(s)} \\
& =\sum_{k=0}^{N+1}\left[\begin{array}{c}
N+1 \\
k
\end{array}\right]^{s} f\left(a q^{k}\right)(-1)^{N+1-k} \beta_{N+1-k}^{(s)}
\end{aligned}
$$


which proves the proposition.

\section{Proposition 2.}

For each $s \in \mathbb{N}_{0}$, the sequence of polynomials $\left(\left(\frac{(x-a)^{(n)}}{[n] !}\right)^{s}\right)$ form a basis of $C\left(V_{q} \rightarrow K\right)$. Each continuous function $f_{.}: V_{q} \rightarrow K$ can be written as a uniformly convergent series

$$
f(x)=\sum_{n=0}^{\infty} c_{n}^{(s)}\left(\frac{(x-a)^{(n)}}{[n] !}\right)^{s} \text { with }\|f\|=\max _{n \geq 0}\left\{\left|c_{n}^{(s)}(q-1)^{n s}\right|\right\}
$$

where $c_{n}^{(s)}=b_{n}^{(s)} /\left((q-1)^{n} q^{n(n-1) / 2} a^{n}\right)^{s}$

Proof:

This follows from the fact that $\frac{(x-a)^{(n)}}{[n] !}=\left\{\begin{array}{l}x \\ n\end{array}\right\}(q-1)^{n} q^{n(n-1) / 2} 0^{n}$.

If we put $s$ equal to one in Proposition 2, we find Jackson's interpolation formula for continuous functions from $V_{q}$ to $K([5])$.

An example.

We have

$$
\begin{aligned}
\beta_{0}^{(s)}= & \beta_{1}^{(s)}=1 \\
\beta_{2}^{(s)}= & {[2]^{s}-1=(q+1)^{s}-1 } \\
\beta_{3}^{(s)}= & {[3]^{s}[2]^{s}-2[3]^{s}+1=\left(q^{2}+q+1\right)^{s}(q+1)^{s}-2\left(q^{2}+q+1\right)^{s}+1 } \\
\beta_{4}^{(s)}= & {[4]^{s}[3]^{s}[2]^{s}-3[4]^{s}[3]^{s}+\frac{[4]^{s}[3]^{s}}{[2]^{s}}+2[4]^{s}-1 } \\
= & \left(q^{3}+q^{2}+q+1\right)^{s}\left(q^{2}+q+1\right)^{s}(q+1)^{s} \\
& -3\left(q^{3}+q^{2}+q+1\right)^{s}\left(q^{2}+q+1\right)^{s}+\left(q^{2}+1\right)^{s}\left(q^{2}+q+1\right)^{s} \\
& +2\left(q^{3}+q^{2}+q+1\right)^{s}-1
\end{aligned}
$$

and after some calculations we find

$$
\begin{gathered}
\left\{\begin{array}{l}
x \\
1
\end{array}\right\}=\left\{\begin{array}{l}
x \\
1
\end{array}\right\}^{2}-q(q+1)\left\{\begin{array}{l}
x \\
2
\end{array}\right\}^{2}+\left(q^{2}+q+1\right)(q+1)^{2} q^{2}\left\{\begin{array}{l}
x \\
3
\end{array}\right\}^{2} \\
-q^{3}\left(q^{3}+q^{2}+q+1\right)\left(q^{2}+q+1\right)\left(q^{4}+3 q^{3}+3 q^{2}+3 q+1\right)\left\{\begin{array}{l}
x \\
4
\end{array}\right\}^{2}+\ldots
\end{gathered}
$$

which gives us a uniformly convergent series. 


\section{References}

1. Y. AMICE, Interpolation $p$-adique, Bull Soc. Math. Fronce $\mathbf{9 2}$ (1964), 117-180.

2. Y. Amice, "Les nombres p-adiques," Presses Universitaires de France, Paris, Collection SUP, Le Mathématicien 14, 1975.

3. S. CAENEPEEL, About p-adic interpolation of continuous and differentiable functions, Groupe d'étude d'analyse ultramétrique ( $Y$. Amice, G. Christol, P. Robba) 25, $9^{e}$ année, 1981/82, 8 p.

4. W. H. SchiкноF, "Ultrametric calculus: An introduction to p-adic analysis," Cambridge University Press, 1984.

5. L. VAN HAMME, Jackson's Interpolation formula in $p$-adic analysis, Proceedings of the Conference on p-adic analysis, report nr. 7806, Nijmegen, June 1978, 119-125.

6. A. C. M. VAN ROOI3, "Non-Archimedean functional analysis," Marcel Dekker, Purc and Applied Mathematics 51, 1978.

Vrije Universiteit Brussel

Faculty of Applied Sciences

Pleinlaan 2

B-1050 Brussels

BELGIUM

Rebut el 2 de Juny de 1993 